\title{
THE STEGANOGRAPHIC SYSTEM FOR HIDDEN TRANSFER OF THE COLOR IMAGES
}

\author{
I. A. Reznik ${ }^{1)}$, R.Kh. Sadykhov ${ }^{2)}$ \\ ${ }^{1)}$ Belarusian State University of Informatics and Radioelectronics, Brovka str. 6, Minsk, 220027, Belarus, \\ i.reznik@inbox.ru \\ ${ }^{2)}$ United Institute of Informatics Problems of NASB, 6, Surganova str., 220012, Minsk, Belarus, \\ rsadykhov@gw.bsuir.unibel.by
}

\begin{abstract}
In the given work the system of the hidden transfer of the graphic information is considered. For hiding the graphic information the digital correlations based on the complex BIFORE transform are used. A crypto stability of a technique is provided by a secret key, with that the hidden image is embedded in the container-image. The problems of efficiency, robustness, accuracy and performance of the suggested method are considered.
\end{abstract}

Keywords: steganography, hiding information, spatial correlator, complex BIFORE (BInary FOurier Representation) transform.

\section{INTRODUCTON}

One of an important direction of the protection from an unauthorized access is a steganography. The steganography is a science about hiding a fact of existing secret information. A digital steganography is based on methods of the digital signal processing. In this work the example of the combining methods of digital steganography with methods of the optical information processing is shown. Use of optical methods gives a good capability for encoding information $[1,2,3,4]$.

The steganographic system for the hidden transfer the color image is proposed. The picture, in which the hidden image is embedded as a rule denominates a container image, and the container with embedded hidden image is the stego-image [5]. A core of our system is two-dimensional spatial correlator, which is used for encoding, embedding and reconstruction of the hidden image. Digital correlations are based on the complex BIFORE (BInary FOurier REpresentation) transform [6]. Note, that classical 2D spatial correlator is based on the complex Fourier transform (CFT). A choice of the complex BIFORE transform (CBT) is conditioned more fast computation performance in comparison with CFT. The secret key of the staganographic system is spatial filter of the correlator.

\section{TWO-DIMENSIONAL SPATIAL CORRELATOR IN BASIS OF COMPLEX BIFORE TRANSFORM}

The two-dimensional spatial correlator consists of three planes. Input plane is described by complex function $a(x, y)=c(x, y) \cdot e^{i \theta(x, y)}$, where $c(x, y)$ is the container image, $\theta(x, y)$ is the phase function of the input domain. The frequency plane is described by key function $K(u, v)=e^{i \phi(u, v)}$, where $\phi(u, v)$ is a random function uniformly distributed on the interval $(-\pi, \pi)[7], K(u, v)$ is a phase-only function. The computational problem is to find such phase function $\theta(x, y)$ to having placed function $a(x, y)$ in input plane, receive in output plane complex function with magnitude that is equal to the hidden image $h(x, y)$. Then output plane is described by the complex function $b(x, y)=h(x, y) \cdot e^{i \psi(x, y)}$, where $\psi(x, y)$ is the phase function of the output domain. Therefore the output correlation function is

$$
b(x, y)=\operatorname{ICBT}[C B T\{a(x, y)\} \cdot K(u, v)] .
$$

From Eq.1, the input function is given by 


$$
a(x, y)=\operatorname{ICBT}\left[C B T\{b(x, y)\} \cdot K^{*}(u, v)\right]
$$

where $K^{*}(u, v)$ denotes complex conjugation to $K(u, v) . \mathrm{CBT}$ and ICBT are the complex BIFORE transform and the inverse complex BIFORE transform, respectively.

CBT belongs to a family of a discrete orthogonal transforms [6]. While the Fourier bases are sinusoids with harmonic frequencies, the BIFORE bases are Walsh functions. Since the Walsh functions are square waves, they take only two values, namely, +1 and -1 . The simplicity of square waves relative to sinusoids allows relatively easy process information [8]. Both CFT and CBT require $N \cdot \log _{2} N$ arithmetical operations, but in case of CFT every arithmetical operation include, beside complex addition, the multiplying.

So, CBT of the function $x(n)$ and its inverse transform ICBT is defined as:

$$
\begin{aligned}
& \{X(n)\}=\frac{1}{N} \cdot[M(n)] \cdot\{x(n)\} \\
& \{x(n)\}=\left[M^{*}(n)\right]^{T} \cdot\{X(n)\}
\end{aligned}
$$

where ${ }^{*}$ and $T$ denotes complex conjugate and transpose, respectively. $[M(n)]$ is matrix of transform, described in [9].

To calculate phase function $\theta(x, y)$ we have used the projection-onto-constraint-sets (POCS) algorithm. The version of this algorithm, optimized for correlation between two images, is described in [10]. The algorithm is ended on $n$-th iteration, when average mean square errors down relatively to average mean square value of an original images less then predefined threshold (in percent). The necessary conditions of the convergence these errors are considered in [7]. This threshold must provide enough approximation magnitude $\left|b_{n}(x, y)\right|$ to the hidden image $h(x, y)$, and magnitude $\left|a_{n}(x, y)\right|$ to $c(x, y)$. Average mean square values of original images are described as:

$$
c_{c p}=\frac{1}{D^{2}} \sum_{x} \sum_{y} c^{2}(x, y),
$$

$$
h_{c p}=\frac{1}{D_{W}^{2}} \sum_{x} \sum_{y} h^{2}(x, y) .
$$

Note that key function $K(u, v)$ is generated once before iterations and it never changes within the iteration process. The block diagram of the POCS algorithm is shown on Fig.1.

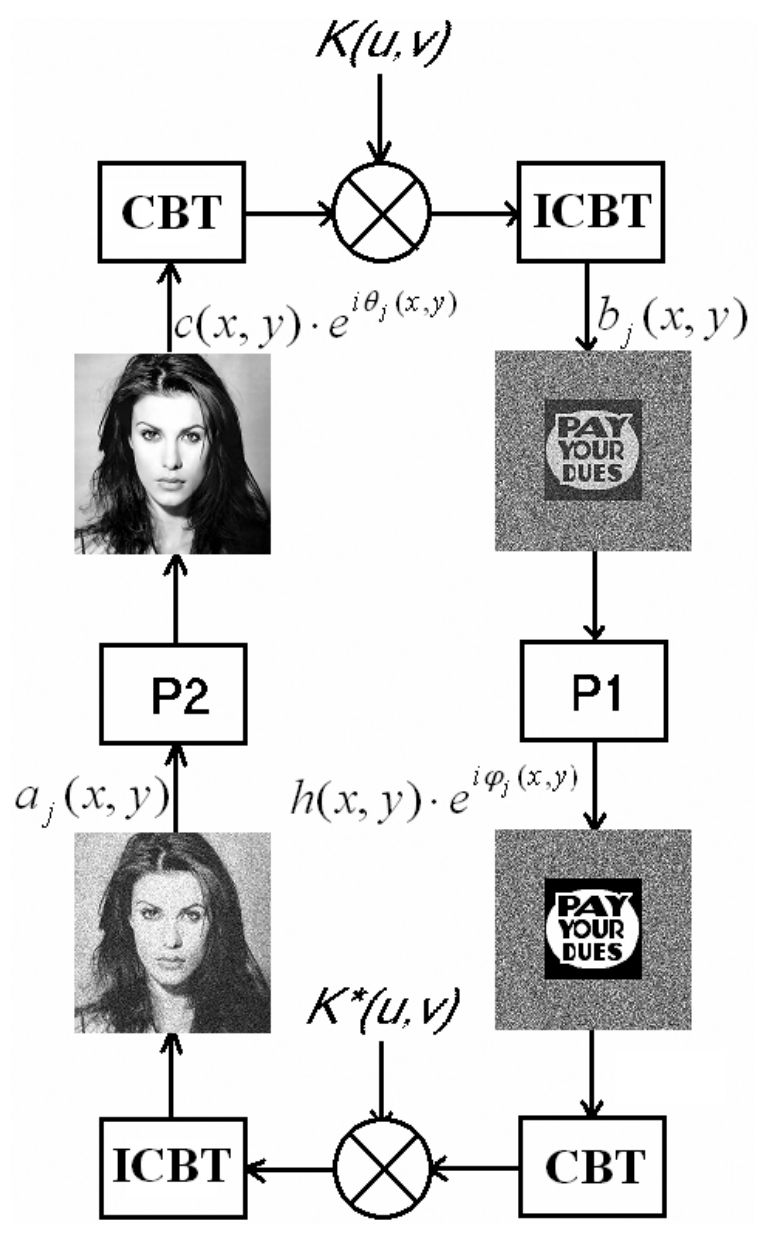

Fig.1 - Block diagram of the POCS algorithm.

\section{Embedding and extracting hidden image}

We grouped respectively color components (red, green and blue) of the container image and hidden image. And then the correlator is learnt for each these pairs. In result of this operations for each color component we have received complex function $a_{n}(x, y)$ described by two linear functions $c_{n}(x, y)$ and $\theta_{n}(x, y)$, where $n$ - count of the iterations spent for training. Now it is required to generate color component of the stego-image on the basis of these two functions.

Let $m$ is a number of bits, required for storage 
every color component. Let the $\operatorname{Ph}(x, y)$ is approximation to the function $\theta_{n}(x, y)$, that was received by quantization on $2^{r}$ levels $(r<m)$, where $r$ is a number of bits for storage the phase function $\theta_{n}(x, y)$. The every color component of the stego-image is constructed by the following algorithm. The brightness of every pixel for each color component equals the brightness respectively pixel of the halftone container image, except for $r$ lower bits. These $r$ lower bits are replaced by value of the function $\operatorname{Ph}(x, y)$. This replacement is possible since modification several lower bits does not heavily distort total picture of the image. Obviously that $r$ must be not more than half $m$. Number of bits used for storage phase, is directly proportional to quality of the reconstructed hidden image, but on the other hand it is inversely proportional to quality of the stego-image. The stego-image that is received in such way we can send by open telecommunication channels.

On the receiving side from each color component stego-image we get two functions $P h(x, y)$ and $c^{\prime}(x, y) . P h(x, y)$ is described by $r$ lower bits of the color component of the stego-image, and $c^{\prime}(x, y)$ is described by $(m-t)$ lower bits. After normalization $P h(x, y)$ and $c^{\prime}(x, y)$ the next correlation is calculated:

$$
b(x, y)=I C B T\left[C B T\left\{c^{\prime}(x, y) \cdot e^{i P h(x, y)}\right\} \cdot K(u, v)\right] .
$$

The magnitude of the received complex function $|b(x, y)|$ is approximately equal to respectively color component of the hidden image $h(x, y)$.

\section{Experimental results}

We have tested the system for three pairs images (hidden and the container- image). As the container and hidden images for all three cases, we used color ( 24 bits per pixel) pictures in the size $512 \times 512$ and $256 \times 256$ of pixels, respectively.

Training of the correlator was stopped, during that moment when the total mean-square error made less than $1 \%$ of average value of the image for every picture (4), (5). The algorithm of training was taken on the average 9 iterations for each color pair. The technique was tested with various count of the bits used for storage of a phase (parameter $r$ - see section 3). Results of experiment are represented on Fig. 2.

During experiment the next parameters have been determined: a) the degree of difference of the transmitted stego-image from the container-image; b) the degree of difference of the extracting hidden image from original hidden image. The measure of difference was defined as average total distinction between all pixels of pair images. Results first pair of images are displayed in Table 1.

Table 1. Degree of difference of pairs images.

\begin{tabular}{|l|r|r|}
\hline \multirow{2}{*}{} & \multicolumn{2}{|c|}{$\begin{array}{c}\text { Number of bits needed for } \\
\text { storage phase }\end{array}$} \\
\cline { 2 - 3 } & $r=3$ & $r=4$ \\
\hline $\begin{array}{l}\text { Stego-image and } \\
\text { container-image }\end{array}$ & $1.7 \%$ & $2.5 \%$ \\
\hline $\begin{array}{l}\text { Extracting image } \\
\text { and hidden image }\end{array}$ & $7.1 \%$ & $4.4 \%$ \\
\hline
\end{tabular}

Besides the basic experiment we have tested the system for stability to losses or purposely damage part of the information by transfer of the stegoimage. Stability of system was checked as follows: in the transmitted image were emulated losses a part $(15 \%, 30 \%$ and $45 \%)$ of pixels - area with zero intensity. The result of experiment is shown on Fig.3.

\section{CONCLUSION}

The steganographic system of the hidden transfer of color pictures optimized on computing complexity is offered. The algorithm of embedding and extracting is based on the modified model of the two-dimensional spatial correlator. Digital correlations are based on complex BIFORE transform. The secret key of stego-system is embedded into the correlator as the spatial filter.

Experiments for three pairs images and various values of parameter $r$ (number of the bits needed for storage of a phase in the transmitted stegoimage) are conducted. Such important qualitative characteristics of system, as a degree of difference of the transmitted stego-image from the original container- image and a degree of difference of the taken hidden image from original hidden image, for various parameters $r$ are calculated. The optimum ratio of quality is shown for a case $r=4$. 
a)
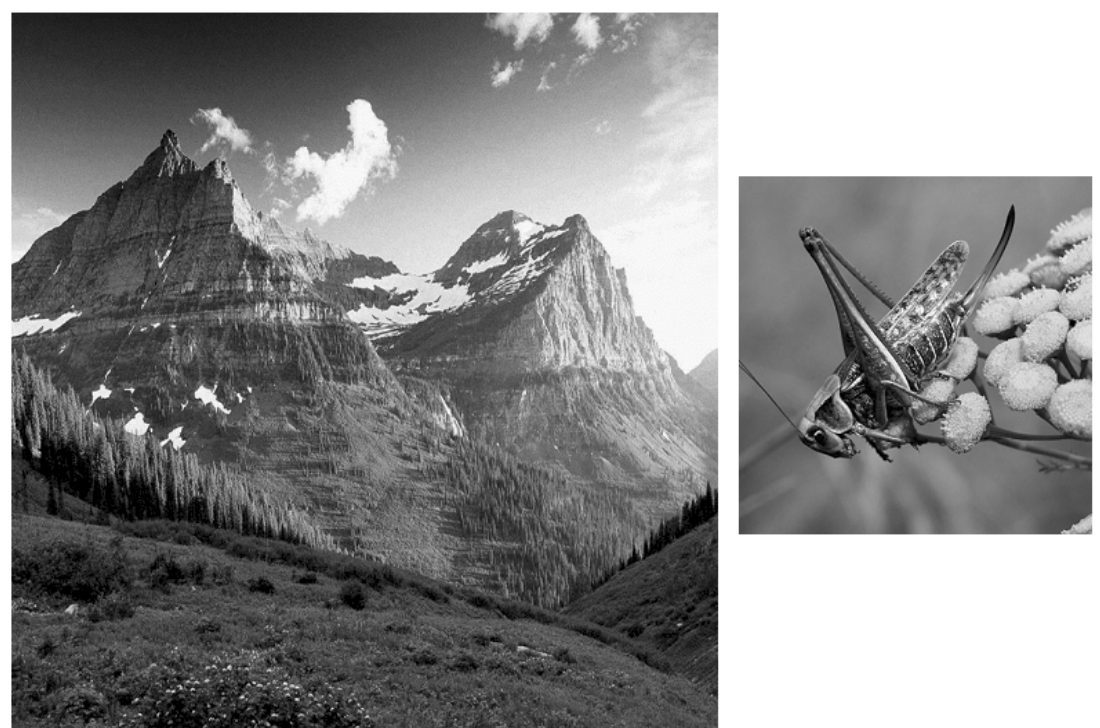

b)
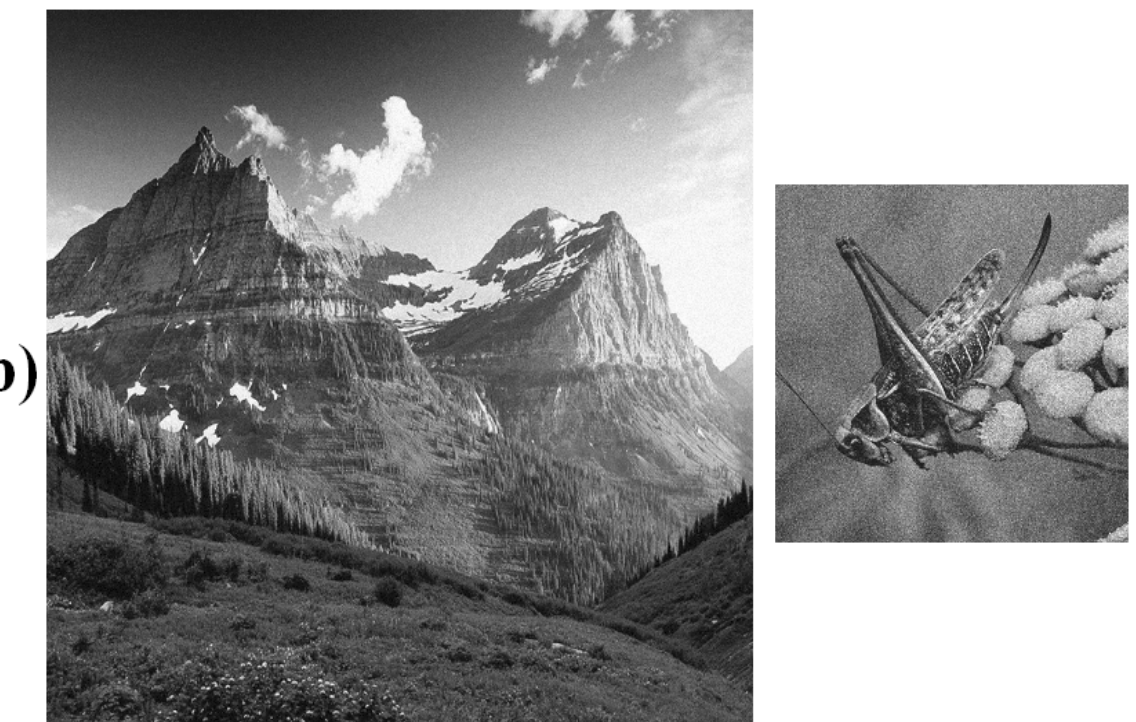

c)
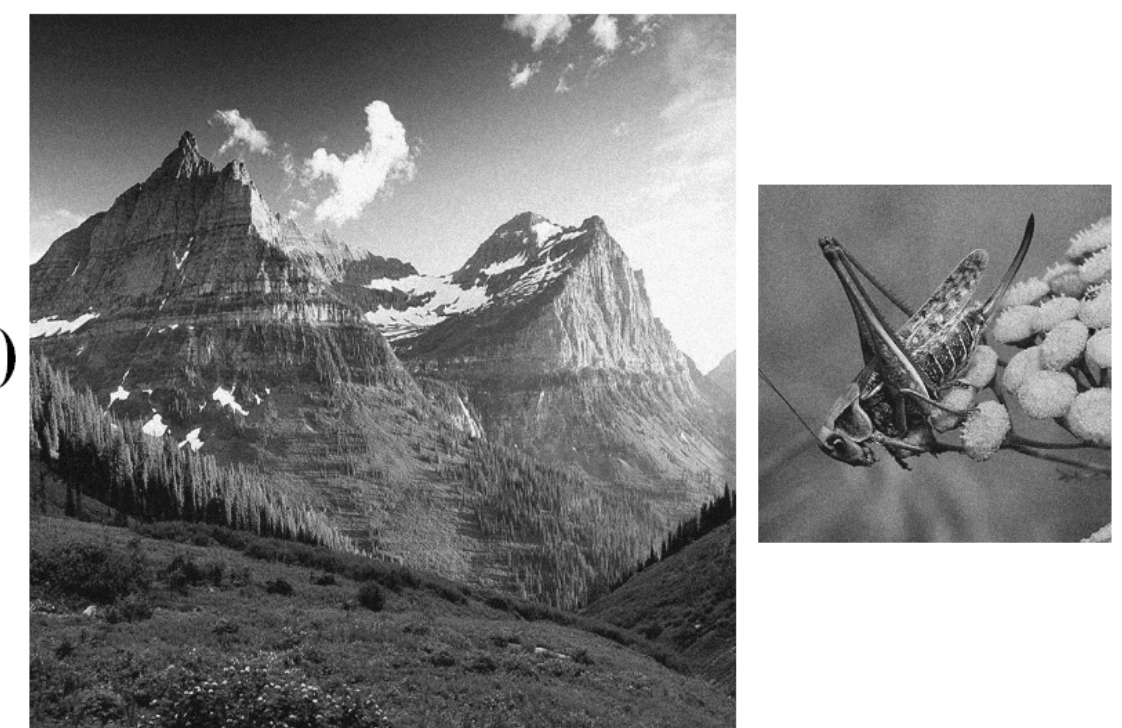

Fig.2 - Result of the experiment:

a) Original container and stego images;

b) Stego image and reconstructed hidden image $(r=3)$;

c) Stego image and reconstructed hidden image $(r=4)$; 
a)
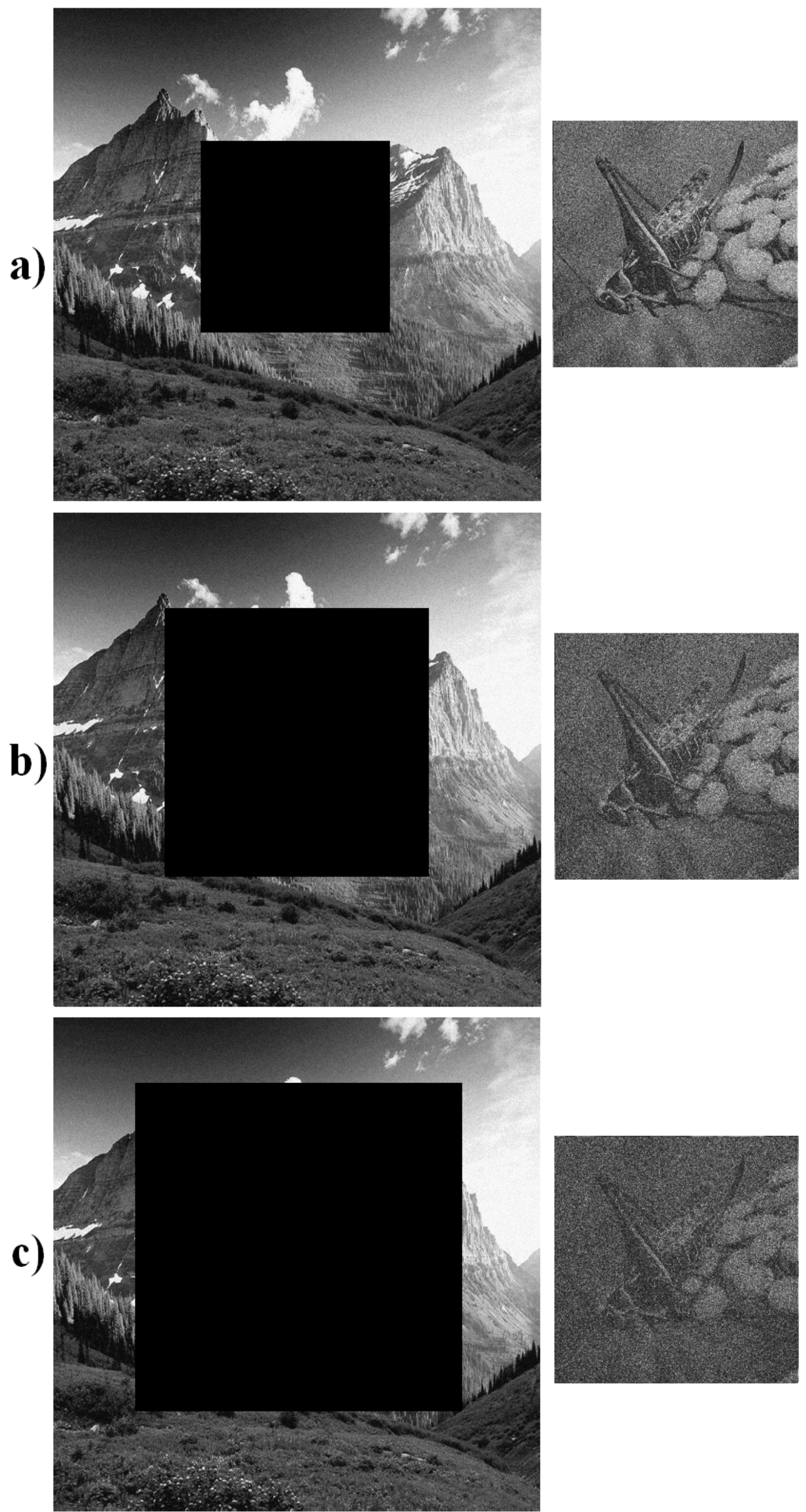

Fig.3. Stego-images with loss a part of pixels and the hidden images taken from them (the number of bits for storage the phase $r=4$ ).

Stability of algorithm to losses or purposely damage of a part of the transmitted information is 
checked up. It is established, that images recognizable even at loss of $30 \%$ of pixels of the stego-image.

Thus, proposed steganographic system allows steadily and safely to transfer color pictures.

\section{REFERENCES}

[1] B. Javidi, "Securing information with optical technologies," Phys. Today 50(3), (1997), pp. 27-32.

[2] B. Javidi and E. Ahouzi, "Optical security system with Fourier plane encoding," Appl. Opt. 37, (1998), 6247-6255.

[3] B. Javidi, L. Bernard, and N. Towghi, "Noise performance of double-phase encryption compared to XOR encryption," Opt.Eng. 38, (1999), 9-19.

[4] S. Kishk, B. Javidi, "Information hiding technique with double phase encoding", Applied Optics (Vol.41, No.26) 10 September 2002, pp.5462-5470.

[5] V.G. Gribunin, „Digital steganography“, St.Petersburg, OOO Solon-Press, 2002.

[6] N. Ahmed, K. R. Rao, R. B. Schultz, "Generalized discrete transform", IEEE Trans. Computing, vol.59, Sept. 1971, pp.1360-1362.

[7] J. Rosen, "Learning in correlators based on projections onto constraint sets," Opt. Lett.18, (1993), pp.1183-1185.

[8] N. Ahmed, K. R. Rao, and A. L. Abdussattar, "BIFORE or Hadamard transform", IEEE Trans. Audio Electroacoust., vol. AU-19, Sept. 1971, pp. 225-234.

[9] N. Ahmed, K. R. Rao, "A decomposition technique for complex N-periodic sequences", IEEE Trans. Audio Electroacoust., Dec. 1971, pp. 324-326.

[10] J. Rosen, B. Javidi, "Hidden images in halftone pictures", Applied Optics (Vol.40, No.20) 10 July 2001, pp.3346-3353.

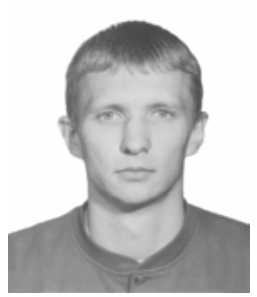

Ivan Reznik was born in Gomel in 1979. I. Reznik, in 1967 graduated from Belarusian State University of Informatics and Radioelectronics on the speciality "Software engineering for information technologies". Now he is a postgraduate student of Computer System Department of BSUIR.

I. Reznik has published several scientific works, including papers and reports at the International Conferences. The area of the scientific investigations includes: steganography, digital signal and image processing, security and encoding systems, artificial neural networks.

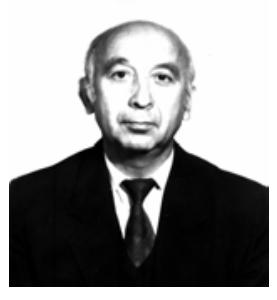

Rauf Kh. Sadykhov, in 1967 graduated from Azerbaijan Polytechnic Institute (Baku) on the specialty "Mathematical and Computing the Instruments and Devices". After graduation from the Institute he attended the postgraduate course at the Institute of Engineering Cybernetics in Minsk. In 1991 he defended his thesis for a scientific degree of a doctor of engineering science in the field of computing science and in 1992 has obtained a professor,s scientific rank.

Since 1995 R.Kh. Sadykhov is a head of Computer System Department in Belarusian State University of Informatics and Radioelectronics and simultaneously he is a head of System Identification laboratory, Institute of Engineering cybernetics of the Belarusian Academy of Sciences. R.Kh. Sadykhov has published more than 350 scientific works, including books, patents, papers and reports at the International Conferences, Symposiums and Workshop. The area of the scientific investigations includes: digital signal and image processing, recognition of handwritten symbol and signature identification, remote-sensing object recognition, computer vision system for the control and recognition, intellectual neural systems, multi-agent systems, parallel architectures for digital signal and image processing.

Prof. R.Sadykhov is the vice-chairman of Belarusian Association of Pattern Recognition (IAPR) and Belarus SIG of International Neural Network Society (INNS), the member of IEE (United Kingdom). 